\title{
edmetic
}

Revista de Educación Mediática y TIC

La competencia mediática en personas mayores

Media competence in the elderly

Fecha de recepción: 21/07/2013

Fecha de revisión: 01/06/2013

Fecha de aceptación: 01/06/2013 


\section{La competencia mediática en personas mayores}

Media competence in the elderly

\section{Isidro Marín Gutiérrez1, Ramón Tirado Morueta² \& Ángel Hernándo Gómez ${ }^{3}$}

\section{Resumen:}

Este artículo ha sido realizado gracias al proyecto I+D+i titulado "La competencia en comunicación audiovisual en un entorno digital" (I+D: EDU 2010-21395-C03) financiado por el Ministerio de Ciencia y Tecnología del Gobierno de España. En ella analizamos el pretest que evaluaba la competencia mediática de personas mayores de 60 años de La Rioja y de Huelva. Los resultados arrojados fueron tenidos en cuenta para el proceso de encuestación que se está realizando actualmente en 10 provincias españolas con una muestra de 615 personas mayores de 60 años. En este artículo estudiaremos el estado de la cuestión en torno a las competencias mediáticas de las personas mayores de 60 años.

Palabras claves: abuelos; competencia comunicativa; tecnología de la información y comunicación y red de información.

\section{Abstract:}

This article was made possible by the R\&D\&i project entitled "Competence in audiovisual communication in a digital environment" (I+D: EDU 2010-21395-C03) funded by the Ministry of Science and Technology, Government of Spain. Here we analyze the pretest that evaluates media competence in citizens aged 60 and over, from La Rioja and Huelva. The results obtained were taken into account for the survey process that is currently being conducted in 10 Spanish provinces with a sample of 615 people over 60 years. This article will look at the current status regarding media literacy of people over 60.

Keywords: grandparents; communicative competence; information technology and communication and information network.

\footnotetext{
1 Universidad Técnica Particular de Loja (Ecuador). imarin1@utpl.edu.ec

2 Universidad de Huelva. rtirado@uhu.es

3 Universidad de Huelva. angel.hernando@dpsi.uhu.es
} 


\section{Introducción}

Actualmente se comenta bastante en los medios de comunicación sobre la llamada "brecha digital". Este concepto hace referencia a la diferencia socioeconómica entre grupos sociales desiguales que tienen acceso a Internet y aquellos grupos que no lo tienen por cuestiones varias. Estas diferencias también se describen en las divergencias sobre las nuevas Tecnologías de la Información y la Comunicación (TIC); como por ejemplo en la utilización de ordenadores, teléfonos móviles de última generación con acceso a Internet de banda ancha, Reproductores de MP4, navegadores GPS U otros dispositivos digitales. Esta brecha digital se basa en diferencias anteriores a este acceso a las nuevas tecnologías. Los términos competencia comunicativa, Tecnología de la Información y Comunicación (TIC), red de información o "brecha digital" va a ser claves para nuestra investigación ya que nos van a indicar las diferencias entre distintos grupos sociales según sea su capacidad para utilizar las TIs de una forma correcta. Van a existir una serie de factores que nos darán esta diferenciación, como puede ser los niveles de alfabetización, carencias de dispositivos, la edad (por eso hemos incluido el término familiar de "abuelos") o problemas de accesibilidad a la tecnología. Muy asociado al término "brecha digital" pero de forma inversa nos encontraremos la expresión "inclusión digital".

Con la "brecha digital" los investigadores sociales establecemos una separación entre regiones e individuos que carecen de los conocimientos técnicos y de las herramientas para el desarrollo de esta nueva sociedad de la información y la comunicación. Como apuntaba el sociólogo Manuel Castells, "una vez que toda la información está en la Red, una vez que el conocimiento está en la Red, el conocimiento codificado, pero no el conocimiento que se necesita para lo que se quiere hacer, de lo que se trata es de saber dónde está la información, cómo buscarla, cómo procesarla, cómo transformarla en conocimiento especifico para lo que se quiere hacer. Esa capacidad de aprender a aprender, esa capacidad de saber qué hacer con lo que se 
aprende, esa capacidad es socialmente desigual y está ligada al origen social, al origen familiar al nivel cultural y al nivel educativo" (Castells, 2005: 212).

La nueva sociedad de la información y de la comunicación necesita nuevas formas de alfabetización digital de toda la sociedad. La utilización y el acceso a la firma digital, el acceso a chats, a foros digitales o a los correos electrónicos son elementos muy importantes para el desarrollo de competencias comunicativas de los ciudadanos (Gros y Contreras, 2006).

Las competencias comunicativas audiovisuales están siendo actualmente estudiadas desde un ámbito académico. Si bien en los medios de comunicación existen constantes alusiones son escasos los artículos y libros que estudian hasta qué punto ciertos grupos sociales se encuentran en esta inclusión digital y cuál es realmente la "brecha digital". Así podríamos descubrir y medir las competencias comunicativas, la red de información o la utilización de las TIC por parte de una sociedad determinada. Revelaremos que la "brecha digital" además de estar relacionada con diferencias socioeconómicas también lo está, y mucho, con la edad, especialmente en grupos de edad más alejadas de las generaciones digitales como son las personas mayores de 60 años. Tampoco se han analizado cómo puede afectar a nuestros abuelos esta "brecha digital" en su cotidianidad del día a día.

El propósito de esta investigación es llegar a poder evaluar sobre competencias comunicativas y mediáticas a las personas mayores para que en futuras investigaciones podamos analizar su nivel de competencias y se puedan elaborar medidas encaminadas a solventar los problemas en este grupo social en concreto.

\section{1. ¿̇Cuál es el concepto de alfabetización mediática?}

La alfabetización mediática la define la Comisión Europea (2009) como la capacidad de consultar, comprender apreciar con sentido crítico y crear 
contenido en los medios de comunicación. Esto es necesario para que se desarrolle una ciudadanía plena y activa. En donde cada persona tenga posibilidades de voz y de voto en decisiones que le atañen. Ofrece a las personas la posibilidad de desarrollarse según sus gustos, formación o ideología sin limitaciones espacio-temporales en la inmensidad de medios de comunicación ligados a la tecnología digital (televisión, cine, Internet, telefonía móvil, radio...).

El término "alfabetización mediática" se ha designado para sugerir una postura de crítica en la evaluación de las noticias que obtenemos de los medios de comunicación de masas. Este concepto da importancia a la recuperación y gestión de la información por parte de los individuos. Paul Gilster lo define como "la capacidad para comprender y utilizar las fuentes de información cuando se presentan a través del ordenador" (Gilster, 1997). Gilster especifica que "la alfabetización digital tiene que ver con el dominio de las ideas, no de las teclas" (Bawden, 2002). Es el conocimiento y la comprensión de lo que uno ve en la pantalla del ordenador, de su teléfono móvil o cuando utiliza un medio en la Red. Uno necesita tener conocimientos de lenguaje audiovisual que se han encontrado presentes anteriormente tanto en radio como en televisión. Gilster afirma: "No sólo debes adquirir la habilidad de encontrar las cosas, sino que además tienes que adquirir la habilidad para utilizar estas cosas en tu vida..." (Bawden, 2002: 395). La persona gracias a esta alfabetización mediática y gracias a Internet va a poder acceder a gran cantidad de información que va a tener que filtrarla según sus intereses pero además va a poder comunicarse, crear y difundir sus propios conocimientos. Gilster (1997) ve en la alfabetización digital el concepto puro de alfabetización que incluía tanto la lectura como la escritura.

\section{2. ¿Cuáles son los enfoques para una alfabetización mediática?}

En los últimos diez años, se han estado desarrollando trabajos que describían y conceptualizaban las habilidades que deberían de tener las personas que 
estaban conectadas en entornos digitales (Cothey, 2002; Hargittai, 2002a, 2002b; Wang, Hawk y YtenopiR, 2000; Zins, 2000).

Pero estos estudios suelen ser muy localizados, centrados en unas competencias muy determinadas y se encuentran limitados principalmente en la búsqueda de información (Marchionini, 1989; Zins, 2000), por lo que no se desarrolla en estudios globales de alfabetización mediática.

Eshet-Alkalai (2004) y también él mismo y su colaborador Ami Chai (2004) han diseñado un nuevo marco conceptual para la alfabetización digital que intenta ser una propuesta práctica. Esta propuesta se dispone de cinco tipos de habilidades de alfabetización, que vislumbra los tipos más importantes de competencias que los individuos utilizan para trabajar de forma eficaz en entornos digitales: a) la alfabetización foto-visual, b) alfabetización de la reproducción, c) la alfabetización informacional, d) la alfabetización hipermedia, y e) la alfabetización socio-emocional.

\subsection{La evaluación de las competencias mediáticas.}

Está existiendo un acuerdo internacional sobre la necesidad de evaluar las competencias mediáticas y conseguir procesos de alfabetización mediática. Organismos internacionales tales como la Comisión de las Comunidades Europeas (2006), la Comisión Europea (2009) o la ONU (2010) están apoyando un desarrollo mundial, coherente y sostenible en educación mediática.

Se han diseñado instrumentos que están consensuados, como por ejemplo el "kit de Educación en Medios" y el "Curriculo de formación del educador en medios y alfabetización comunicativa" de la UNESCO, la Carta Europea para la Alfabetización Mediática y el apoyo de la Alianza de las Civilizaciones en la Educación en Medios, mediante congresos y publicaciones. La Declaración de Grunwald (en 1982), la Proclamación de Alejandría acerca de la Alfabetización Informacional y el Aprendizaje de por Vida (en 2005), así como la Agenda de París para la Educación en Medios (en 2007) nos indican líneas estratégicas y futuras investigaciones sobre este tema 
(Frau-Meigs y Torrent, 2009: 10).

España no es ajena a estos cambios mundiales, durante los últimos años se han producido cambios en este sentido. Juan Ferrés (2006) junto a un equipo de expertos elaboraron un documento titulado "Competencias en comunicación audiovisual", con una sistemática aproximación al concepto y las dimensiones de "competencia comunicativa", definida como la "capacidad de un individuo para interpretar y analizar desde la reflexión crítica las imágenes y los mensajes audiovisuales, y para expresar con una mínima corrección en el ámbito comunicativo. Esta competencia está relacionada con el conocimiento de los medios de comunicación y con el uso básico de las tecnologías multimedia necesarias para producirla" (Ferrés, 2006: 10).

\subsection{Los programas universitarios de Aula de Mayores}

Desde la década de los años 60 del siglo pasado en los Estados Unidos y posteriormente en la década de los años 70 del siglo pasado en el contexto europeo se han ido creando programas para personas mayores (Orte, 2006). Estas políticas y programas orientados a la población mayor no llegaron a España hasta la década de los años 90 del siglo pasado en donde se crearon programas universitarios para mayores. Uno de estos proyectos fue el de la Universidad de Alicante, llamado Proyecto AEPUMA (2007), en donde se ofrecían cursos para personas mayores de 50 años. Los contenidos de cada universidad para este alumnado especial son diferentes. Dichos planes de estudios tienen una validez académica oficial pero sin eficacia profesional puesto que no son títulos oficiales (Lorenzo, 2008).

Los beneficios que tienen estas personas mayores son, según Arnay (2006), la consecución de más recursos cognitivos, sociales y afectivos para el desarrollo personal, afrontar nuevos retos, tener una actitud reflexiva y crítica de la sociedad en donde se encuentran. Estas personas mayores, nuestros abuelos, solicitan estos cursos, que les puedan ofrecer una formación profunda 
y amplia, que les entusiasme y les motive, para tener mayores relaciones sociales y también para que reivindiquen su status vital.

Estos programas universitarios de Aulas de Mayores suponen un reto para las universidades españolas ya que este colectivo está aportando beneficios a la comunidad universitaria. Es necesario que se les garantice una buena formación científica pero no hay que olvidar la formación humana. Además deben desarrollar distintas competencias y habilidades individuales para su desarrollo. Para tal fin, las propuestas y prácticas de cada currículum de estas personas deben tener en cuenta la contextualización, sus necesidades, demandas y posibilidades de mejorar este colectivo (Ricoy, 2004).

Esta nueva sociedad del conocimiento obliga a las universidades, según Vázquez y Pérez (2006), a acercar a las personas mayores a las TIC. Varias investigaciones apuntan a las ventajas de su utilización en la educación de personas mayores (Barrio, Barrio y Quintanilla, 2007; Cano y Expósito, 2006; De arriba, 2008). Pero no se trata de formarlos en el manejo de las herramientas sino en las utilidades para su vida diaria, como pedir una cita médica, realizar gestiones bancarias, localizar a un antiguo familiar o llamar a un amigo. En otras palabras, es necesario formarlos en competencias mediáticas para su desarrollo personal y social.

Lo importante es que las personas mayores tengan la oportunidad de interpretar y analizar, desde su criterio, las imágenes y los mensajes audiovisuales. Les tenemos que ceder expresarse con una mínima corrección en el ámbito comunicativo (Ferrés, 2006). Las personas mayores han de ser capaces de realizar un análisis crítico de los productos audiovisuales que ven y escuchan. Que sean capaces de interactuar con ellos de forma eficaz (Ferrés, 2007).

\section{Objetivos}

El objetivo de este artículo es el proceso de pilotaje de un instrumento para la 
evaluación de las competencias mediáticas en personas mayores de 60 años que participan en los cursos de para personas mayores de la Universidad y compararlos con personas que no acuden a las Aulas de Mayores de las universidades españolas. Esta investigación está enmarcada en el estudio financiado en la Convocatoria I+D del Ministerio de Ciencia e Innovación con clave: EDU2010-21395-C03-03. Y para ello se toma como punto de referencia el documento-base mencionado anteriormente en el que se van a recoger las siguientes dimensiones (Ferrés y Aguaded, 2011):

- Estética: Esta dimensión va a evaluar la capacidad de analizar y valorar los diferentes mensajes audiovisuales que tienen los encuestados, desde el punto de vista de la innovación formal, la temática y las relaciones que establecen con otras formas de manifestación mediática y artística.

- Lenguaje: El lenguaje comprende el conocimiento de los códigos que consiguen el lenguaje audiovisual así como también la capacidad de utilizarlos para comunicarse. También se refiere a la capacidad de análisis de los mensajes audiovisuales desde la perspectiva de la significación, de las estructuras narrativas y de las categorías y géneros (Ferrés, 2006).

- Ideología y valores: Permite la capacidad de lectura comprensiva y crítica de los mensajes audiovisuales que son portadoras de ideologías y valores. También se refiere al análisis crítico de los citados mensajes (Ferrés, 2006).

- Recepción y audiencia: Aquí se analizan los conocimientos de los encuestados sobre el proceso de recepción de los mensajes audiovisuales. Sobre sus conocimientos sobre cómo se miden las audiencias.

- Tecnología: Esta dimensión hace referencia a los conocimientos de los encuestados sobre el manejo de las herramientas tecnológicas sencillas que se encuentran en la comunicación audiovisual.

- Producción y programación: En esta última dimensión se evalúa el grado de conocimiento sobre las rutinas productivas y sobre la organización y funcionamiento de las unidades emisoras de mensajes audiovisuales. 


\section{Metodología de la prueba piloto}

Se elaboró el cuestionario inicial revisando los objetivos de la investigación y con las Recomendaciones de la Comisión Europea del 20 de agosto del 2009 "sobre la alfabetización mediática en el entorno digital para una industria audiovisual y de contenidos más competitiva y una sociedad del conocimiento incluyente". Posteriormente se comenzó a revisar otras investigaciones que estuvieran basadas en la construcción de instrumentos de medida de la competencia mediática en la ciudadanía.

Tras la revisión de otras encuestas se realizó una matriz en donde se asociaban una serie de indicadores en cada una de las competencias que comprendía el listado de referencia sobre la base de seis dimensiones que vamos a analizar: lenguaje, tecnología, recepción-interacción, producción, ideología y valores y, finalmente, estética.

Se revisaron otros instrumentos que tuvieran los mismos objetivos que el nuestro y se desarrolló la triangulación de juicios en cuanto a la asignación de ítems e indicadores, sobre cada una de nuestras competencias.

Se creó el primer cuestionario provisional que fue sometido a una prueba piloto sobre una muestra de personas mayores de 60 años en el entorno de la Universidad de La Rioja. Sobre la base de este primer cuestionario se creó un segundo cuestionario sobre una muestra de personas mayores de 60 años en el Aula de Mayores de la Universidad de Huelva. Tras esta última comprobación el cuestionario está listo para poder aplicarse a una muestra representativa para analizar las competencias mediáticas de las personas mayores.

Actualmente se está realizando el proceso de encuestación hasta el 31 de marzo del 2013 en diez provincias españolas (La Rioja, Córdoba, Murcia, Málaga, Lugo, Sevilla, Granada, Valencia, Cantabria y Huelva) tanto en grupos del Aula de la Experiencia como en grupos fuera de la universidad. La muestra seleccionada es de 615 sujetos alcanzando la cifra a 18 de marzo del 2013 los 609 cuestionarios totales. 
A continuación mostraremos un análisis breve de los resultados de la segunda prueba piloto que sirvió de referencias para la construcción del instrumento final que se está aplicado en estos momentos. Los resultados en profundidad de la primera prueba piloto se publicaron en la revista Iconol4 (Tirado Morveta, Hernando Gómez, García Ruiz, Santibáñez Velilla y Marín Gutiérrez, 2012).

\section{SUJETOS Y PRIMEROS RESULTADOS}

En la primera prueba piloto realizada en la muestra de La Rioja con personas mayores de 60 años se seleccionó una muestra atendiendo a las siguientes variables: género, nivel de estudios finalizados con los siguientes resultados:

a) En cuanto al género, la muestra de La Rioja tiene 10 mujeres y 7 hombres.

b) En cuanto al nivel de estudios la muestra está formada por: 6 cuestionarios realizados por encuestados con estudios primarios, 6 cuestionarios realizados por encuestados con estudios secundarios y 5 cuestionarios realizados por encuestados con estudios universitarios.

c) Todos los entrevistados están jubilados y los ingresos oscilan ente 600 euros y 2.400 euros.

d) Todos los participantes conviven en sus hogares con familiares.

e) Ninguno de los encuestados con estudios primarios han recibido formación en comunicación audiovisual.

f) Entre los entrevistados con estudios secundarios la mayoría afirma haber asistido a talleres, conferencias o cursos y formación especializada.

g) En cuanto se refiere a los encuestados con estudios universitarios, tres de ellos responden haber asistido a talleres, conferencias o cursos y formación especializada y el resto confiesa no haber recibido formación en comunicación audiovisual.

En la segunda prueba piloto realizada en la muestra de Huelva con personas mayores de 58 años se seleccionó una muestra atendiendo a las siguientes variables: género, nivel de estudios finalizados con los siguientes 
resultados:

a) En cuanto al género, la muestra de Huelva tiene 15 mujeres y 6 hombres.

b) En cuanto al nivel de estudios la muestra está formada por: 3 cuestionarios realizados por encuestados sin estudios, 13 cuestionarios realizados por encuestados con estudios primarios, 5 cuestionarios realizados por encuestado con estudios secundarios y 1 cuestionarios realizados por entrevistados con estudios universitarios.

c) En cuanto a su situación profesional 14 están jubilados, 3 son empresarios, 2 trabajan en empresas privadas y 2 son empleados públicos. Se eliminó la pregunta sobre el nivel de ingresos.

d) En la muestra de Huelva 6 personas viven solas, 10 viven solo con su pareja y 6 viven con más de dos personas.

e) Ninguno de los encuestados sin estudios han recibido formación en comunicación audiovisual. Y de los que tienen estudios primarios 5 no han recibido formación y 8 sí han recibido alguna formación audiovisual.

f) En cuanto se refiere a los encuestados con estudios universitarios, el único de ellos responde haber asistido a talleres, conferencias o cursos y formación especializada.

\section{Conclusiones}

Como resultado de los dos estudios pilotos presentados se construyó finalmente una versión definitiva para el estudio tomando en cuenta las siguientes consideraciones generales:

1. El nivel de alfabetización mediática de los mayores es considerablemente bajo si se tienen en cuenta el listado de competencias de partida. Principalmente en la muestra de Huelva.

2. No obstante, ello debe interpretarse en el contexto de una concepción crítica de la competencia. En otras palabras, el listado de competencias propuesto responde a un enfoque ingenuo, por lo que sería conveniente realizar estudios que permitan identificar referentes atendiendo a las 
identidades de los colectivos a evaluar.

3. Existen competencias, que debido al escaso o nulo nivel de respuesta sobre el indicador se han suprimido de la versión definitiva. Principalmente el bajo nivel de respuesta se ha dado en la muestra de La Rioja, ya que, si comprobamos el número de preguntas en la Tabla 1, el cuestionario de La Rioja contaba con 36 preguntas sobre competencias. Cuya duración era aproximadamente de una hora. Mientras que el cuestionario de Huelva tenía 22 preguntas. Y tenía una duración de media hora.

4. En consecuencia, el número de ítems sobre las dimensiones se disminuye de 36 a 22, tratando de esta forma que su cumplimentación sea más fácil y rápida, atendiendo a las sugerencias de las personas que lo cumplimentaron, tanto en La Rioja como en Huelva. La sensación de cansancio, y más en este tipo de personas, puede tener efectos que desvíen la fiabilidad de las respuestas.

5. Se ha tratado de evitar en el segundo borrador de cuestionario los ítems de doble entrada (matrices) para facilitar la respuesta.

6. El número de ítems, tras el segundo recorte en el cuestionario de Huelva ha dejado de ser numeroso y responde a las exigencias de la investigación que trata de averiguar el nivel de competencia mediática atendiendo a las seis dimensiones presentadas en la Tabla 1.

\begin{tabular}{c|c|c} 
& $\begin{array}{c}\text { Cuestionario pasado } \\
\text { en La Rioja }\end{array}$ & $\begin{array}{c}\text { Cuestionario pasado } \\
\text { en Huelva }\end{array}$ \\
\hline$N^{\circ}$ de ítems de la dimensión Lenguaje & 7 & 3 \\
\hline$N^{0}$ de ítems de la dimensión Tecnología & 4 & 3 \\
\hline$N^{\circ}$ de ítems de la dimensión Producción & 9 & 3 \\
\hline $\begin{array}{c}\text { No de ítems de la dimensión } \\
\text { Recepción/Interacción }\end{array}$ & 4 & 4
\end{tabular}




\begin{tabular}{c|c|c}
$\begin{array}{c}\text { No de ítems de la dimensión } \\
\text { Ideología/Valores }\end{array}$ & 7 & 4 \\
\hline$M^{\circ}$ de ítems de la dimensión Estética & 5 & 5 \\
\hline $\begin{array}{c}\text { Total de ítems por dimensiones } \\
\text { Tabla 1: Número de preguntas por dimensiones en cada uno de los cuestionarios } \\
\text { Fuente: Elaboración propia }\end{array}$
\end{tabular}

\section{Referencias bibliográficas}

AEPUMA (2007). Los programas universitarios para mayores en España: Análisis y evaluación de las enseñanzas universitarias para mayores en España y Europa. Alicante: Universidad de Alicante. Recuperado de: http://www.imsersomayores.csic.es/documentos/docu-mentos/bruprogramas-01.pdf.

ARNAY, J. (2006). Las necesidades socieducativas de las personas mayores en la sociedad actual. (pp. 135-173). En C. Corte (coord.). El aprendizaje a lo largo de toda la vida. Los programas universitarios de mayores. Madrid: Editorial Dykinson.

BARRIO, J.L., BARRIO, M.L. y QUINTANILLA, M. (2007). Tecnología y educación de adultos. Cambio metodológico en las matemáticas. Revista Complutense de Educación, 18 1), 113-132.

BAWDEN, D. (2002). Revisión de los conceptos de alfabetización informacional y alfabetización digital. Anales de Documentación, 5, 361-408.

CANO, P. Y EXPÓSITO, R. (2006). Una propuesta para el uso de nuevas tecnologías como herramientas docentes en los programas universitarios para personas mayores. (pp. 315-323). En M. C. Palmero (coord.). Formación universitaria de personas mayores y promoción de la autonomía personal. Políticas socioeducativas, metodológicas e innovaciones. Burgos: Universidad de Burgos.

CASTELLS, M. (2005). Internet y la sociedad red. (pp. 203-228). En D. de Moraes (Coord.). Por otra comunicación. Los media, globalización cultural y 
poder. Barcelona: Icaria editorial.

COMISIÓN DE LAS COMUNIDADES EUROPEAS (2006). Comunicación de la Comisión Europea: La nueva generación de programas comunitarios de educación y formación después de 2006. Recuperado de http://www.sifemurciaeuropa.com/servlet/sife.GestionDocs?METHOD=VISTAGESTIONDOCS.

COMISIÓN EUROPEA (2009). Study on Assessment Criteria for Media Literacy Levels, Bruselas.

Recuperado

de http://ec.europa.eu/culture/media/literacy/docs/studies/eavi study ass ess crit media lit levels europe finrep.pdf.

COTHEY, V. (2002). A longitudinal study of world wide web users' informationsearching behavior. Journal of the American Society for Information Science and Technology, 53(2), 67-78.

DE ARRIBA, J.A. (2008). Aprendiendo a resolver casos reales mediante la utilización de herramientas informáticas de aprendizaje y colaboración. Estudio experimental en un contexto de formación universitario. Revista de Universidad y Sociedad del Conocimiento (RUSC), 5(2), 36-49. Recuperado de http://www.uoc.edu/rusc/5/2/dt/esp/arriba.html

ESHET-ALKALAI, Y. (2004). Digital literacy: A conceptual framework for survival skills in the digital era. Journal of Educational Multimedia and Hypermedia, 13(1), 93-106.

ESHET-ALKALAI, Y. Y AMI CHAI-HAMBURGER (2004). Experiments with digital literacy. Cyber Psychology, 7(4), 425-434.

FERRÉS, J. (2007). La competencia en comunicación audiovisual: dimensiones e indicadores. Comunicar, 29, 100-107.

FERRÉS, J. Y\& AGUADED, J. I. (2011). Competencia mediática. Investigación sobre el grado de competencia en la ciudadanía en España. ITE. Ministerio de Educación y Ciencia.

FERRES, J. (2006). La competencia en comunicación audiovisual: propuesta articulada de dimensiones e indicadores. Quaderns del CAC, 25, 9-17. 
Recuperado

de

http://www.cac.cat/pfw files/cma/recerca/quaderns_cac/Q25ferres2 Es.pdf

FRAU-MEIGS, D. y TORRENT, J. (2009). Políticas de educación en medios: Hacia una propuesta global. Comunicar, 32, 10-14.

GÁZQUEZ, J.J. y PÉREZ, M.C. (2006). El reto de la disponibilidad, formación y uso de las nuevas tecnologías en los mayores universitarios. (pp. 325-331). En M.C. Palmero (coord.). Formación universitaria de personas mayores y promoción de la autonomía personal. Políticas socioeducativas, metodológicas e innovaciones. Burgos: Universidad de Burgos.

GILSTER, P. (1997). Digital Literacy. New York: Wiley Computer Publishing.

GROS, B. y CONTRERAS, D. (2006). La alfabetización digital y el desarrollo de competencias ciudadanas. Revista Iberoamericana de Educación, 42, 103-125.

HARGITTAI, E. (2002a). Beyond logs and surveys: Indepth measures of people's web use skills. Journal of the American Society for Information Science and Technology, 53(14), 1239-1244.

HARGITTAI, E. (2002b). Second-level digital divide: Differences in people's online skills. First Monday, 7(4). Recuperado de http://firstmonday.org/issues/issue7 4/hargittai/index.html

LORENZO, J. A. (2008). Propuesta de regularización del acceso a las enseñanzas universitarias oficiales para mayores de 50 años desde los programas universitarios para personas mayores. (pp. 151-164). En M. C. Palmero (coord.). Formación universitaria de personas mayores y promoción de la autonomía personal. Políticas socioeducativas, metodológicas e in-novaciones. Burgos: Universidad de Burgos.

MARCHIONINI, G. (1989). Information-seeking strategies of novices using a fulltext electronic encyclopedia. Journal of the American Society for Information Science, 40(1), 54-66.

NACIONES UNIDAS (2010). Objetivos de Desarrollo del Milenio. Informe 2010. 
Recuperado

de:

www.un.org/es/comun/docs/?path=/spanish/millenniumgoals/pdf/MDG Report_2010_SP.pdf.

ORTE, C. (COORD.) (2006). El aprendizaje a lo largo de toda la vida. Los programas universitarios de mayores. Madrid: Editorial Dykinson.

RICOY, M. C. (2004). Claves para identificar el perfil del alumnado de la educación básica de adultos. Enseñanza, 22, 283-300.

TIRADO, R., HERNANDO, A. GARCÍA, M.R., SANTIBÁÑEZ, J. y MARÍN, I. (2012). La competencia mediática en personas mayores. Propuesta de un instrumento de evaluación. Icono 14, 10(3), 134-158. Recuperado de http://www.iconol4.net/ojs/index.php/iconol4/article/view/211

WANG, R., HAWK, W. B. y YTENOPIR, C. (2000). Users' interactions with World Wide Web resources: An exploratory study using a holistic approach/Information. Processing and Management, 36(2), 229-251.

ZINS, C. (2000). Success, a structured search strategy rationale, principles and implications. Journal of the American Society for Information Science, $51(13), 1232-1247$.

\section{Cómo citar este artículo:}

Marín Gutiérrez, I., Tirado Morveta, R. y Hernándo Gómez, Á. (2013). La competencia mediática en personas mayores. EDMETIC, Revista de Educación Mediática y TIC, 2(2), 95-111. 